\title{
Visualizing Strain Evolution and Coordinated Buckling within CNT Arrays by In Situ Digital Image Correlation
}

\author{
Matthew R. Maschmann, Gregory J. Ehlert, Sei Jin Park, David Mollenhauer, \\ Benji Maruyama, A. John Hart, and Jeffery W. Baur*
}

Spatial mapping of strain fields within compressed carbon nanotube (CNT) array columns is achieved using digital image correlation (DIC) analysis of in situ scanning electron microscopy (SEM) image sequences. Full-field displacement and strain maps are generated based upon the motion of the constituent CNTs, which serve as a traceable high-contrast speckle pattern for DIC analysis. The deformation modes and CNT array buckling characteristics vary systematically as a function of column aspect ratio, including bending, crushing, and bottom-up buckle accumulation behaviors. In spite of disparate appearing deformation modes, strain maps indicate that CNT array buckling consistently initiates at $5 \%$ local principal strain $\left(\varepsilon_{2}\right)$ for all columns. The ability to quantitatively assess the deformation modes and buckling behavior of CNT arrays at the nanoscale will enable their improved design for highstrain electrical contacts, compliant thermal interfaces, force sensors, energyabsorbing foams, or other applications.

\section{Introduction}

The mechanical behavior of carbon nanotube (CNT) arrays (also called vertically aligned CNTs or forests) under compressive loading is receiving significant scientific attention because of the complex multi-scale mechanics as well as the importance of mechanical properties to diverse applications. The performance

Dr. M. R. Maschmann, Dr. D. Mollenhauer,

Dr. B. Maruyama, Dr. J. W. Baur

Air Force Research Laboratory

Materials and Manufacturing Directorate

AFRL/RX, Wright Patterson Air Force Base,

OH 45433, USA

E-mail: Jeffery.Baur@wpafb.af.mil

Dr. M. R. Maschmann

Universal Technology Corporation

Beavercreek OH, 45432, USA

Dr. G. J. Ehlert

Department of Mechanical and Aerospace Engineering

University of Florida

Gainesville, FL, 32611-6250, USA

S. J. Park, Prof. A. J. Hart

Mechanosynthesis Group

Department of Mechanical Engineering

University of Michigan

Ann Arbor, MI, 48109, USA

DOI: $10.1002 / \mathrm{adfm} .201200676$ of CNT arrays in applications such as therma ${ }^{[1-3]}$ and electrical ${ }^{[4,5]}$ interface materials, deformable CNT array strain biosensors, ${ }^{[6]}$ and electrical brushes ${ }^{[7]}$ rely intimately on array mechanics for proper operation. Often, optimization for a specific application requires consideration of two or more coupled properties, such as stiffness and damping, or stiffness and thermal conductivity. However, the means by which global deformation of a CNT array generates localized deformation of the constituent CNTs, and how this, in turn, determines the collective mechanical properties is not thoroughly understood.

Many studies have examined the mechanical properties of CNT arrays via nanoindentation $^{[8-11]}$ or uniaxial compression, ${ }^{[12-15]}$ and showed that entangled arrays locally yield by coordinated buckling of CNTs. The buckles propagate in a direction perpendicular to CNT alignment, and strain recovery after unloading can be significantly elastic ${ }^{[12,15]}$ or plastic ${ }^{[10,11,13,14]}$ based on the diameter, alignment, and packing density of the constituent CNTs. Buckle initiation has been observed preferentially near the top ${ }^{[11]}$ or bottom side of an array, ${ }^{[12-14]}$ but it is has not been shown how the initiation of buckling relates to the geometry and global deformation of the arrays. More recently, in situ scanning electron microscopy (SEM) mechanical testing has enabled direct observation of buckle evolution and has been utilized for the uniaxial compression of CNT array columns ${ }^{[13]}$ and the nanoindentation of CNT arrays. ${ }^{[11]}$ A continuum-based analytical model inspired by the in situ CNT array column compression observations ${ }^{[13]}$ was recently used to predict the strain distribution that arises during buckling; ${ }^{[16]}$ however, an experimental technique capable of mapping local strain fields within CNT arrays to validate this and other models, and to relate mechanical properties to CNT morphology and array geometry, has not yet been reported. Likewise, a means to quantify the seemingly diverse CNT buckling behavior at a local level is needed to better understand the mechanics driving buckle formation, and to provide a basis for comparing experimental results from different studies.

In order to map strain fields within CNT arrays, a highresolution, non-contact method is needed, because the small size scale of CNT arrays prevents spatially resolved mechanical measurements via attached hardware such as strain gages. One such 
option, digital image correlation (DIC), is frequently employed to analyze the full-field deformation mechanics of macroscale objects, including low density cellular materials such as open-cell foam. ${ }^{[17-20]}$ DIC utilizes a software algorithm to track the translation of a high contrast and randomly distributed speckle pattern applied to the surface of a deforming body. The incremental displacements of discrete points on the patterned surface are tracked by analyzing the relative motion of pixel grayscale intensity patterns. ${ }^{[21]}$ Application of a suitable speckled surface pattern is often an intensive process, requiring a random distribution of sufficiently high contrast features throughout the surface area of interest. While application of a surface pattern is suitable for mechanical testing of macroscale objects, the addition of highcontrast particles for the purpose of DIC could significantly alter the inherent mechanics of nanoscale material systems.

Perhaps because of these surface preparation requirements, DIC has not yet been used extensively for in situ SEM testing of micro and nanoscale structures, particularly for full-field analysis. To date, DIC has been employed to generate 1-D displacement maps of a $70 \mathrm{~nm}$ diameter $\mathrm{Au}$ nanowhisker under tension, ${ }^{[22]}$ vector displacement maps of a $1 \mu \mathrm{m}$ diameter NiTi pillar ${ }^{[23]}$ during bending, and full-field displacement and strain maps of a Au nanoparticle decorated $50 \times 10 \mathrm{~mm} \mathrm{Al}$ foil during thermal expansion. ${ }^{[24,25]}$ For the $\mathrm{Au}$ nanowhisker and NiTi pillar experiments, naturally-occurring, yet highly localized high-contrast regions allowed for localized DIC analysis but did not provide an adequate speckle pattern for computing a complete spatial deformation map of the deforming bodies. Most recently, DIC of an individual deformed single-walled CNT was achieved at the atomic level using aberration corrected HRTEM imaging. For this experiment, the individual atoms within the CNT provided the requisite pattern for deformation analysis. Results for a single CNT indicated significant internal shear effects that were successfully described by Timoshenko beam theory, ${ }^{[26]}$ but the impact of these findings to arrays of multiwalled CNTs is unclear.

We demonstrate the application of DIC to the in situ SEM compression of CNT array columns. The inherent high contrast pattern generated by the CNTs near the visible surface of the CNT array act as a traceable speckle pattern and allows direct analysis by DIC without surface modification. The technique enables observation of microscale deformation mechanics while elucidating the strain and displacement fields with nanoscale spatial resolution. By a rigorous study of the deformation of lithographically defined CNT array columns with various dimensions, we reveal three significantly different column deformation modes that depend on the column geometry and determine the location of initial buckling, Despite these differences, spatially resolved strain maps of the CNT columns reveal a consistent local CNT array buckle initiation criterion of $5 \%$ minor principal strain $\left(\varepsilon_{2}\right)$, which is independent of column dimensions.

\section{Results and Discussion}

\subsection{Validation of DIC for CNT Arrays}

CNT array columns with square cross sections were grown by chemical vapor deposition on lithographically patterned catalyst films, and were compressed in a customized micromechanical test frame ${ }^{[10]}$ utilizing flat punch indentation tips. Further details may be found in the Experimental section. The matrix of tested column dimensions include widths of 10,30 , and $100 \mu \mathrm{m}$ with nominal heights of 25,50 , and $75 \mu \mathrm{m}$, achieved by changing the CNT growth time. In situ SEM compression testing was conducted utilizing sufficient magnification and image resolution to achieve pixel dimensions between $10-40 \mathrm{~nm}$, which is comparable to the CNT diameters within the array $(8-10 \mathrm{~nm})$. The square cross section of the columns presented a planar face for SEM observation and allowed the entirety of the surface to remain within the focal plane of the SEM. These imaging conditions enabled observation of CNT interactions and assessment of the relative motion of CNTs during column deformation. Representative images demonstrating the typical level of detail captured by these imaging conditions, including enlarged insets to show pixel dimensions relative to CNT feature sizes, may be found in the Supporting Information Figure S1.

The continuity of the CNT entanglement within the array during compression is an important factor relative to the quality of DIC. For reliable image correlation, the speckle pattern established by the interconnected CNTs should remain cohesive during DIC analysis. Therefore, the contact points established between neighboring CNTs must be sufficiently strong to maintain the interconnected structure of the array during deformation. The potential breaking and re-establishment of CNT-to-CNT contacts would alter the speckle pattern, resulting in correlation anomalies or a lack of correlation. The extent to which CNT contact points within an array remain rigidly affixed during deformation is not fully understood and is essential for the generation of a reliable speckle pattern for the DIC technique. While inspection of CNT entanglement at the relatively low magnification imaging conditions appropriate for observation of column deformation shows no signs of CNT re-arrangement during deformation due to broken neighbor contacts, high magnification observation of a deforming CNT array was utilized to examine the behavior of CNT contact points in greater detail.

A $30 \mu \mathrm{m}$ wide by $75 \mu \mathrm{m}$ tall CNT array column was compressed and observed in situ at $50000 \times$ magnification. Because of the reduction in the field of view at high magnification, only a limited area of the column is observed. Figure 1a shows an SEM image of the entire CNT array column, while Figure 1b displays frames from the compression sequence obtained at the central bottom region of the column obtained at $50000 \times$ magnification. The interconnected nature of the CNTs is clearly visible at high magnification (Figure 1b), with the array composition resembling that of an open-cell foam. ${ }^{[10-13]}$ The initial high magnification frame displays the undeformed array morphology, while the subsequent images are obtained at sequentially increased levels of global applied strain. The CNT-to-CNT contacts are remarkably stable, even during the significant deformation, bending, and local cellular buckling. This sequence shows that individual CNTs within the array behave similarly to struts in a cellular solid, pivoting and deforming about their fixed contact points with neighboring CNTs. As a guide, three representative CNT contact points are circled in Figure $1 \mathrm{~b}$ and are tracked throughout the deformation sequence. By evaluating the change in distance between the points, relative strains in excess of $60 \%$ are observed between the points during the 

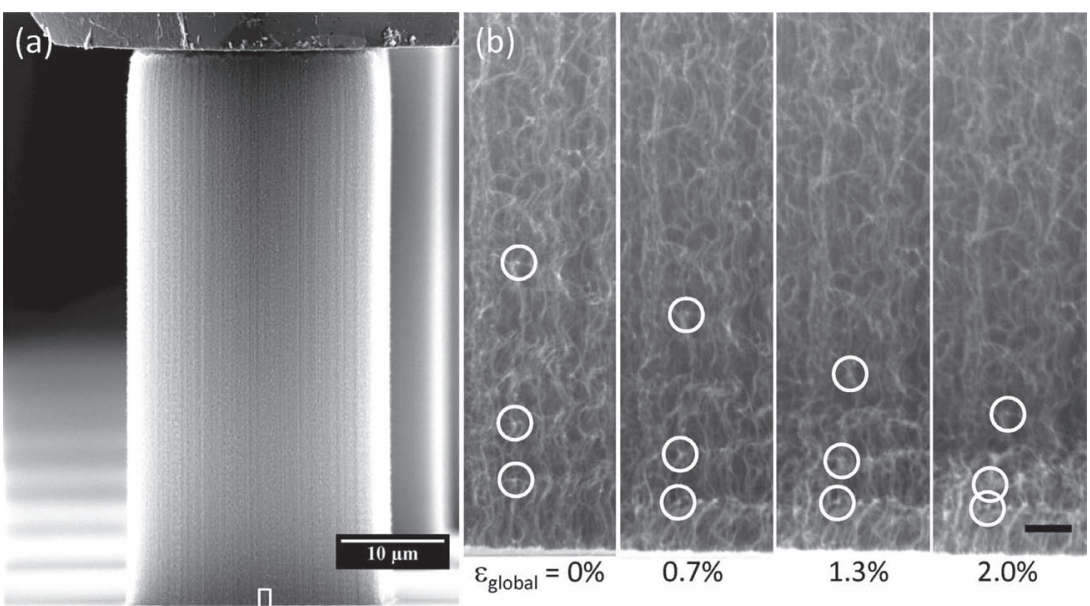

Figure 1. To investigate the stability of CNT-to-CNT contacts, a $30 \mu \mathrm{m}$ wide, 75 tall $\mu \mathrm{m}$ CNT array column, shown in (a), was compressed at $50000 \times$ magnification, with an approximate viewable region outlined by the box near the growth substrate. b) Sequential SEM images of the compressed CNT array column at $50000 \times$ magnification demonstrate the stability of CNT contacts, even at greater than $60 \%$ local compressive strain. Scale bar $=500 \mathrm{~nm}$. A video of the full deformation sequence may be viewed in Supporting Information Video S1.

deformation sequence to $2 \%$ global strain, with little evidence of broken contacts. The rigidity of CNT contacts is even more readily observed in the animated deformation sequence found in the Supporting Information Video S1. The stability of CNT contact points suggests that CNT array entanglement remains intact during significant compressive deformation, creating a coherent speckle pattern that is suitable for reliable tracking of CNTs using conventional DIC algorithms.

Having demonstrated the continuity of the CNT array morphology, validation of 2-D DIC was examined using in situ SEM compression sequences of deforming columns. We emphasize that two-dimensional DIC requires no external calibration for strain measurement, as deformation is determined by comparing each deformed image to an initial undeformed image. However, to confirm proper operation of the analysis and to further validate $2 \mathrm{D}$ deformation assumptions, the spatial averages of the DIC-computed values of $\varepsilon_{\mathrm{yy}}$ (vertical strain) and $\varepsilon_{2}$ (minor principal strain) were compared to the global applied strain to ensure consistency. The vertical strain and minor principal strain are defined as

$\varepsilon_{Y Y}=\frac{\partial v}{\partial y}$

$\varepsilon_{2}=\left(\frac{\varepsilon_{x x}+\varepsilon_{Y Y}}{2}\right)-\sqrt{\left(\frac{\varepsilon_{x x}+\varepsilon_{Y Y}}{2}\right)^{2}+\varepsilon_{x y}^{2}}$

where $v$ represents vertical displacement, $\gamma$ is the vertical coordinate, $\varepsilon_{x x}$ is strain in the horizontal orientation, perpendicular to and in plane with $\varepsilon_{y y}$, and $\varepsilon_{x y}$ is the in-plane shear stress.

Two columns representing distinctly different deformation modes were selected for the validation experiment. These columns include a $30 \mu \mathrm{m}$ wide, $25 \mu \mathrm{m}$ tall column (exhibiting crushing behavior) and a $30 \mu \mathrm{m}$ wide, $75 \mu \mathrm{m}$ tall column (exhibiting bottom-up buckle accumulation behavior). Global strain is defined as the vertical displacement of the indenter head relative to the undeformed CNT array column height and is measured using a virtual extensometer within the DIC software. Likewise, spatial strain averages are computed by the DIC software at each global strain evaluation point. Importantly, the initial contact between the indenter tip and a column ( $0 \%$ global strain) is defined by the first detectable vertical displacement field established within the column. This initial contact is established between the indenter tip and a small number of asperities at the top surface of the column (for example, Figure S2a,b Supporting Information). Due to the non-idealized surface texture at the nanoscale and the inherent heterogeneity of the CNT array morphology, a uniform strain field is not observed, even at the smallest global strains examined $(<0.01 \%)$. Full area contact is established at approximately $1.75 \%$ global strain (440 $\mathrm{nm}$ displacement) for the $25 \mu \mathrm{m}$ tall column and approximately $0.3 \%$ global strain $(200 \mathrm{~nm}$ displacement) for the $75 \mu \mathrm{m}$ tall column. The comparison of global strain and spatially averaged strain fields is ceased shortly after initiation of buckling for each column, as significant deformation and localized folding within the buckling region leads to a loss of localized image correlation.

It is important to note that a correlation error exceeding the maximum allowable confidence interval ( 0.1 pixels) leads to a localized loss of correlation. Data in these areas (observed as localized gaps in map coverage) is omitted from the strain maps by the DIC software, while analysis for the remainder of the column surrounding these areas remains unaffected. Loss of correlation occurs most frequently in and around a buckled CNT array region, as significant deformation and inward folding during buckle formation prevent accurate correlation. Because correlation is increasingly lost near a buckle (the regions of highest localized strain), the reported average strain is artificially low after buckling and is no longer a valid representation of the global strain state of the column. Despite loss of local correlation, DIC maintains the strain field in the non-buckled portion of the array, enabling visualization of how the strain is transmitted to the area of high deformation, and how high local deformation affects the strain field in the rest of the column.

As observed in Figure 2a,b, global strain increases linearly at a rate consistent with the input displacement rate. Due to incomplete area contact between the indenter tip and the CNT array columns, however, the magnitude of spatially averaged $\varepsilon_{\mathrm{yy}}$ and $\varepsilon_{2}$ fields lags that of global strain throughout the tests. Upon full area contact between the indenter and the column, a linear average strain rate is obtained for $\varepsilon_{y y}$ and $\varepsilon_{2}$ as a function of global strain for both columns, as observed in Figure 2a,b. Solid lines with a slope of unity, representing the global strain rate, have been overlaid to the $\varepsilon_{\mathrm{yy}}$ and $\varepsilon_{2}$ spatial average data for comparison. For each column, the rate of change in average $\varepsilon_{\mathrm{yy}}$ and $\varepsilon_{2}$ agree well with the global strain application rate.

The contour maps of $\varepsilon_{\mathrm{yy}}$ and $\varepsilon_{2}$ observed in Figure 2c,d demonstrate the highly non-uniform distribution of the strain within the CNT array columns. These strain maps are obtained at the 

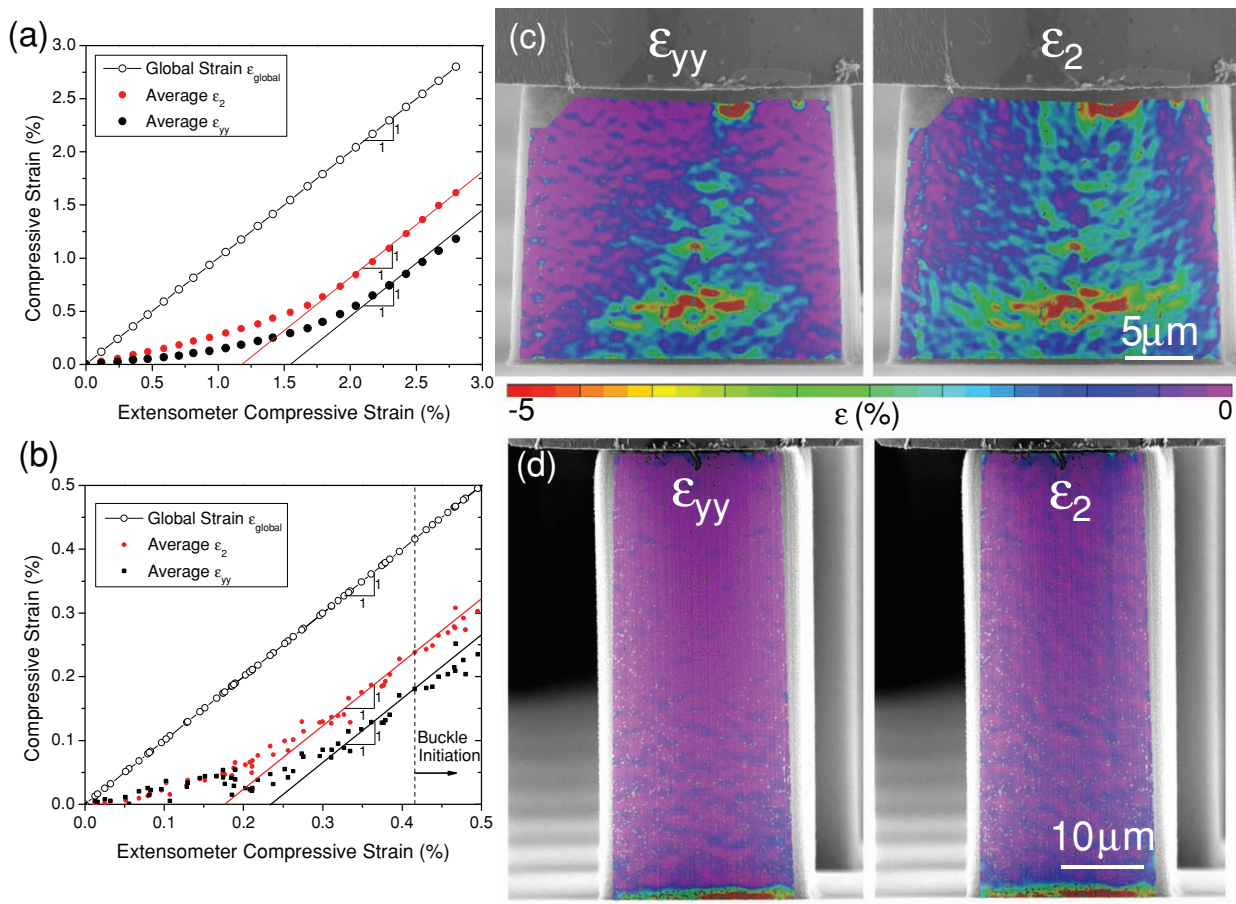

Figure 2. Comparison of applied global strain to the spatially averaged strain fields measured by DIC. Plots of the global strain (measured from first contact), average $\varepsilon_{y y}$ and average $\varepsilon_{2}$ for a uniaxially compressed a) $30 \mu \mathrm{m}$ wide, $25 \mu \mathrm{m}$ tall and b) $30 \mu \mathrm{m}$ wide, $75 \mu \mathrm{m}$ tall CNT array column demonstrate a linear average strain rate upon full area contact. Solid lines represent a slope of unity, consistent with global strain application rate. Contour maps of $\varepsilon_{y y}$ and $\varepsilon_{2}$ obtained at the maximum global strain reported in (a,b) demonstrate highly non-uniform strain distributions for the c) $25 \mu \mathrm{m}$ tall and d) $75 \mu \mathrm{m}$ tall CNT array columns, respectively.

greatest global strain utilized for the spatial averaging experiment $(2.5 \%$ and $0.5 \%$ global strain for the $25 \mu \mathrm{m}$ and $75 \mu \mathrm{m}$ tall columns, respectively). The greatest local strain is found in a lateral band located below the midsection of the $25 \mu \mathrm{m}$ tall column and near the bottom of the $75 \mu \mathrm{m}$ tall column. Additionally, the contour maps further demonstrate that the magnitude of $\varepsilon_{2}$ is slightly greater than that of $\varepsilon_{\mathrm{yy}}$, particularly away from the bands of strain concentration. The previously discussed consistency between the global strain rate and the spatial averages of $\varepsilon_{\mathrm{yy}}$ and $\varepsilon_{2}$, particularly in light of the highly non-uniform spatial distribution of strain, indicates excellent correlation and further validates the use of 2D DIC analysis relative to CNT array columns.

It is important to also consider SEM imaging artifacts such as electron beam rastering, image drift, and image distortion. Image drift is inherently present in SEM imaging, and beam rastering inconsistencies may lead to image distortions between subsequent image scans. These artifacts were examined by imaging a CNT array column using SEM imaging conditions that were identical to those used for mechanical testing. A series of 100 images was obtained over the span of approximately $2.5 \mathrm{~h}$. As discussed in the Supporting Information and observed in Figure S2, image drift in the vertical orientation, parallel to the column axis, varied from 5.77-6.53 pixels along the length of the column after 2.5 hours. Strain artifacts due to image drift and distortion were in the range of $-0.2 \%-0.1 \%$. Similar results have been observed by others after 30 minutes of SEM scan time. ${ }^{[25,27,28]}$ Because the applied strains examined in this study far exceed these sources of error, image drift and distortion are negligible and do not adversely affect our results.

\subsection{DIC Analysis of In Situ CNT Array Column Compression}

A systematic study examining the influence of aspect ratio (defined as column height divided by width) of CNT array columns subjected to uniaxial compression was explored. The column deformation behavior, and the location and appearance of CNT buckling varied considerably, yet systematically, for the 9 column aspect ratios examined. In this study, 2-5 columns of each nominal aspect ratio were compressed. We found three deformation modes: bending, at the highest examined aspect ratios (2.6-6.0); crushing, at the lowest aspect ratios (0.2-0.8); and bottom-up buckle accumulation, for intermediate aspect ratios (0.6-2.7). We observe that CNT array columns with similar dimensions always deform in a similar manner. Moreover, the strain fields of similarly deforming columns share similar characteristics. To more fully examine the deformation modes and the role of strain relative to CNT array behavior, each deformation mode is described in detail with representative SEM compression sequences and strain maps.

The metrics of vertical displacement $(\mathrm{V})$, horizontal displacement $(U)$, vertical strain $\left(\varepsilon_{\mathrm{yy}}\right)$, horizontal strain $\left(\varepsilon_{\mathrm{xx}}\right)$, shear $\left(\varepsilon_{\mathrm{xy}}\right)$, and minor principal strain $\left(\varepsilon_{2}\right)$ were examined via DIC for each column. To further illustrate the typical outputs of the technique, DIC output from the analysis of a $30 \mu \mathrm{m}$ wide, $25 \mu \mathrm{m}$ tall CNT array column is discussed in the Supporting Information and displayed in Figure S3. While the full set of output variables provides additional insight into the deformation mechanics of CNT array columns that is unattainable by SEM evaluation performed by the unassisted eye, further analysis will focus most 
heavily on $\varepsilon_{2}$, as we find that this parameter provides the most consistent and relevant data with respect to the critical deformation behavior. The magnitude and behavior of $\varepsilon_{2}$ is similar to that of $\varepsilon_{\mathrm{yy}}$ (see Figure $2 \mathrm{c}, \mathrm{d}$ ); however, $\varepsilon_{2}$ corrects for experimental alignment errors between the test frame and the y-axis of evaluation for DIC and includes compressive contributions of off-axis strains. Unless otherwise stated, further discussions regarding local strain quantities will, therefore, be discussed in terms of $\varepsilon_{2}$.

\subsection{Bending of High Aspect Ratio Columns}

The compressed columns in our testing with the highest aspect ratio (2.6-6.0) exhibited significant bending, similar in appearance to the response of slender columns to uniaxial compression. Specifically, these include each $10 \mu \mathrm{m}$ wide column, regardless of height. Because of their relatively small widths, the $10 \mu \mathrm{m}$ wide columns possess a second moment of inertia that is approximately 81 times less than the $30 \mu \mathrm{m}$ wide columns, and 10000 times less than the $100 \mu \mathrm{m}$ wide columns. Consequently, these columns offer bending stiffness values that are considerably smaller than the wider columns assuming similar moduli, making them significantly less resistant to bending in response to mechanical moments. As with all columns, deformation is plastic after CNT array buckling, with little recovery observed after retraction of the indenter tip.

Figure 3 displays deformation sequences of two representative bending columns, including a nominally $75 \mu \mathrm{m}$ tall
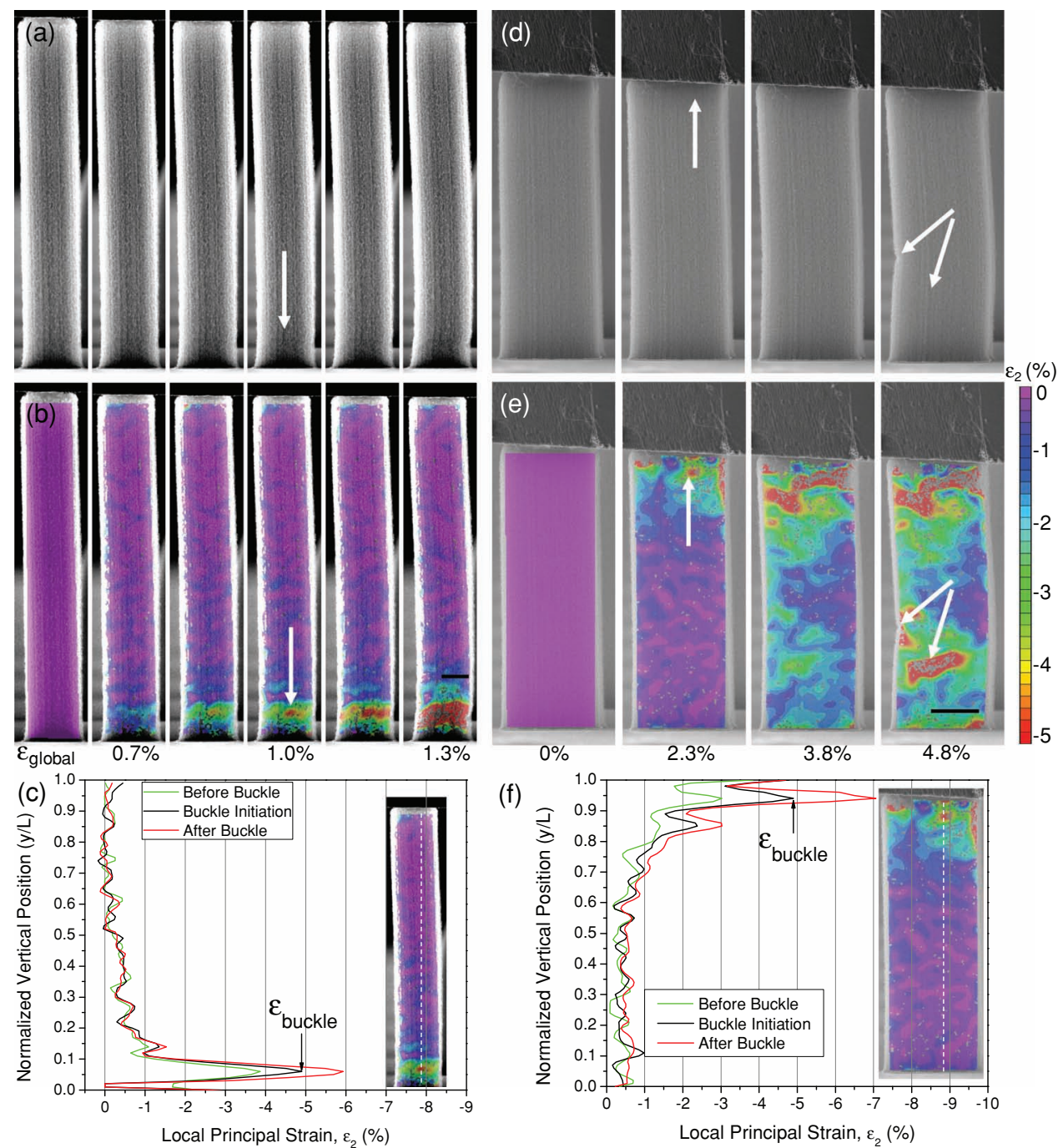

Figure 3. Bending behavior of a-c) $10 \mu \mathrm{m}$ wide, $75 \mu \mathrm{m}$ tall CNT array column and d-f) $10 \mu \mathrm{m}$ wide, $25 \mu \mathrm{m}$ tall CNT array column. SEM sequences reveal that the a) $75 \mu \mathrm{m}$ tall experiences its most significant bending about its midsection, while the d) $25 \mu \mathrm{m}$ tall column bends about its midsection. Strain $\left(\varepsilon_{2}\right)$ maps $(b, e)$ show non-uniform strain distribution throughout compression and buckle formation from regions of local strain maxima. Strain $\left(\varepsilon_{2}\right)$ profiles obtained about a vertical line intersecting the location of buckle formation (c,f) reveal that CNT buckling initiated at approximately $5 \%$ strain for each column. Arrows denote buckle initiation sites. Scale bars $=5 \mu \mathrm{m}$. Videos of the SEM deformation sequences and strain $\left(\varepsilon_{2}\right)$ evolution for the columns may be found in Video S2 and S3 in the Supporting Information. 
(Figure 3a) and a 25 tall $\mu \mathrm{m}$ (Figure 3d) column having a width of $10 \mu \mathrm{m}$. Bending columns with nominal heights of 50 and $75 \mu \mathrm{m}$ generally develop a prominent buckle near their base under compression, similar to behavior depicted Figure 3a, while $25 \mu \mathrm{m}$ tall columns develop multiple buckles, similar to the behavior depicted in Figure 3d. During compression of the nominally $75 \mu \mathrm{m}$ tall column, localized bending and CNT array buckling are observed near the base of the column, particularly at $1 \%$ global strain and greater. At approximately $1 \%$ global strain, the column develops a pronounced buckle near its base. The buckled region propagates laterally with increased global strain and acts as a hinge for further angular rotation. The $25 \mu \mathrm{m}$ tall column bends about its midsection, rather than its base. CNT buckling first initiates near the top surface at approximately $2.3 \%$ global strain in response to bending of the column from left to right. Further global strain leads to additional bending and the formation of a kink and additional CNT buckling near the column midsection at $4.8 \%$ global strain.

Strain maps generated from the deformation sequences, illustrated in Figure 3b, clearly illustrate a localization of strain and the evolution of CNT array buckling. The nominally $75 \mu \mathrm{m}$ tall column exhibits a lateral band of enhanced strain located near the bottom of the column that develops at low global strain and grows in width and magnitude with increased global strain. The maximum strain within the band is approximately 5 times greater than the global applied strain. Outside of the lateral band, strain is negligible for a majority of the column height. Buckle initiation at $1.0 \%$ global strain occurs within the central portion of the strain band, where local strain is greatest. The local strain at the region of buckle initiation is approximately $5 \%$. In fact, as the buckle propagates laterally, the local strain near the leading edges of the propagating buckle is also approximately $5 \%$ strain. The area around the buckle at or exceeding $5 \%$ local strain expands rapidly after buckle initiation, likely a result of the column locally folding inward about the buckle. Strain outside of the buckled region remains significantly less than $5 \%$.

The $25 \mu \mathrm{m}$ tall column initially accumulates strain in a band near its top surface, consistent with the area of initial buckle formation, as previously discussed. Figure 3d-e depicts buckle formation from within the region of enhanced local strain at approximately $2.3 \%$ global strain. By $3.8 \%$ global strain, the top buckle, identifiable as a thin line of lost correlation (lack of color), has spread across the width of the column. As with the $75 \mu \mathrm{m}$ tall column, buckle initiation and propagation is again observed at approximately 5\% local strain. After the full lateral propagation of the initial top buckle, strain distributes across the entire length of the column. Strain on the left side of the column is generally greater than that on the right side, as the bending moment imparted by the column deformation adds additional stress to the left side of the column while reducing stress on the right side of the column. As the column yields about its midsection at $4.8 \%$ global strain, a strain concentration and CNT array buckling event is observed on the left (concave) side of the column while strain at the right (convex) side of the bending column is negligible at the same height. Again, initiation of this second buckle occurs at approximately 5\% local strain, while strain away from the buckling region is significantly less than $5 \%$. Videos S2 and S3 in the Supporting Information display the deformation sequences and $\varepsilon_{2}$ evolution of the $10 \mu \mathrm{m}$ wide, $75 \mu \mathrm{m}$ tall CNT array column and the $10 \mu \mathrm{m}$ wide, $25 \mu \mathrm{m}$ tall CNT array column depicted in Figure 3, respectively.

To more clearly quantify the local strain in and around the regions of buckle initiation, strain profiles are generated about vertical lines spanning the length of the column and intersecting the area of buckle initiation. Profiles of the strain field are evaluated about the same vertical line for the sequential frame immediately before buckle initiation, the frame of initiation, and the frame immediately after initiation. The position of the vertical lines utilized for strain evaluation are displayed as dashed lines in Figure 3c,f. For both columns, buckle initiation occurs at $4.9 \%$ local strain, suggesting a potential consistent buckle initiation criterion. Furthermore, local strain rapidly exceeds 5\% in the buckling region as additional global strain is added after buckling. Local strain in the buckling region increases from approximately $3.9 \%-6.0 \%$ during the $0.2 \%$ change in applied global strain for the $75 \mu \mathrm{m}$ tall column (Figure 3c). Similarly, local strain in the buckled region increased from 3.0\%-7.2\% for the $25 \mu \mathrm{m}$ tall column for a $0.4 \%$ change in applied global strain (Figure 3f). In both cases, minimal changes in the local strain are observed far from the buckling region. While initiation of the first CNT buckle of the $25 \mu \mathrm{m}$ tall column is evaluated and displayed in Figure 3f, a similar local strain of approximately $5 \%$ is observed for the initiation of the buckles near the midsection of the column at approximately $4.8 \%$ global strain.

Significant out-of-plane translation and rotation of high aspect ratio bending columns is observed after buckle formation (Figure 3 and Videos S2 and S3 in the Supporting Information). For 2D DIC, both effects may lead to an undesired distortion of the observed strain fields. The out-of-plane translation contributes strain distortion proportionally to the translation distance divided by the effective object distance from the image capturing lens. ${ }^{[21,29]}$ Assuming out-of-plane motion of up to $10 \mu \mathrm{m}$ and an object distance of $1 \mathrm{~cm}$, out-of-plane translation contributes minimal error $(<0.1 \%)$ to the reported strain values. Out-of-plane rotation, however, can significantly distort 2D DIC strain analysis, with associated strain distortions represented by

$\varepsilon_{x x} \approx-\frac{Y \sin \theta}{Z}$

$\varepsilon_{y Y} \approx \cos \theta-1-\frac{Y \sin 2 \theta}{Z}$

where $Y$ represents the length of the angled section, $\theta$ is the out-of-plane rotation angle, and $Z$ is the object working distance from the system optics. The large working distance relative to the column distance makes distortion of $\varepsilon_{\mathrm{xx}}$ negligible. However, modest angular distortion may lead to significant $\varepsilon_{\mathrm{yy}}$ distortion for the bending columns. As an example, an out-ofplane rotation of $20^{\circ}$ is sufficient to produce a $\varepsilon_{\text {yy }}$ distortion of $6 \%$ (compression). Because the rotation angle associated with bending columns may be excessive, variable, and directionally indeterminate, quantitative DIC evaluation for bending columns is ceased shortly after buckle formation.

As will be discussed, the crushing and bottom-up buckling accumulation mechanisms exhibited by lower aspect ratio columns do not suffer from global out-of-plane distortions to 
the same degree as bending columns. Angular distortion is generally low for these aspect ratios at the column level $\left(<5^{\circ}\right)$, particularly prior to CNT buckling. However, within the localized buckling region substantial rotation $\left(>45^{\circ}\right)$ due to inward folding during buckle formation may greatly distort the observed strain by factors expressed in Equation 3 and 4. Strain outside of the localized buckling region will remain free of such distortions. As a result, local strain values reported by the 2D analysis within a buckled region are likely exaggerated, while the strain computation in surrounding regions are relatively free of angular and out-of-plane displacement distortions.

\subsubsection{Crushing of Low Aspect Ratio Columns}

In contrast to the bending behavior previously observed for the highest aspect ratio columns, the lowest aspect ratio columns (0.2-0.8) buckle near their midsection upon sufficient loading in a manner resembling crushing of thin walled containers (Figure 4a). The columns exhibiting crushing behavior include $25 \mu \mathrm{m}$ tall columns of 30 and $100 \mu \mathrm{m}$ width. These columns show little evidence of bending before buckling, and maintain a nearly vertical neutral axis. Crushing columns accommodate significantly more global strain prior to buckle formation (3-5\%), presumably because of their relatively wide base and short stature. Buckling may initiate and propagate rapidly along the width of a crushing column with little incremental global strain. A total of 2-4 sequential buckles often form prior to the onset of CNT array densification, whereby the entirety of a column is enveloped into the buckled region.
The in situ compression sequence and strain evolution of a $100 \mu \mathrm{m}$ wide, 25 tall $\mu \mathrm{m}$ crushing column is shown in Figure $4 \mathrm{a}-\mathrm{c}$. Prior to buckling, the column appears to deform uniformly in response to the compressive load. However, the corresponding strain maps show that strain is non-uniformly distributed, with significant strain concentrated in a lateral band near the vertical midsection of the column. Strain outside of the band is diminished, though generally non-zero. Strain within the band builds as global strain is added to the column. Buckle initiation is evident at $4.3 \%$ global strain, with the buckle measuring approximately $15 \mu \mathrm{m}$ in width within the first frame of buckle observation ( $0.1 \%$ global strain increments). Consistent with bending columns, the local strain at the leading edge of the buckle is again approximately $5 \%$, while strain within the buckle exceeds $5 \%$. A vertical profile of the strain field (Figure 4c) near the leading edge of the buckle more clearly demonstrates a local strain of approximately 5\%. Local strain in the buckled region increases from approximately $2.5-8.2 \%$ in only $0.2 \%$ change in global strain during buckle formation (Figure 4c). Figure $4 \mathrm{~b}$ shows significant strain relaxation away from the buckling region after full buckle propagation across the column face at $6.2 \%$ global strain. Strain relaxation behavior away from the buckled region may also be observed in Figure 4c during the initial stages of buckle formation and propagation. Similar crushing deformation and buckling behavior is observed for $30 \mu \mathrm{m}$ wide, $25 \mu \mathrm{m}$ tall CNT array columns, as observed in Figure 2c. Video S4 in the Supporting Information displays the deformation sequence and $\varepsilon_{2}$ evolution of the $100 \mu \mathrm{m}$ wide, $25 \mu \mathrm{m}$ tall CNT array column depicted in Figure 4a,b.
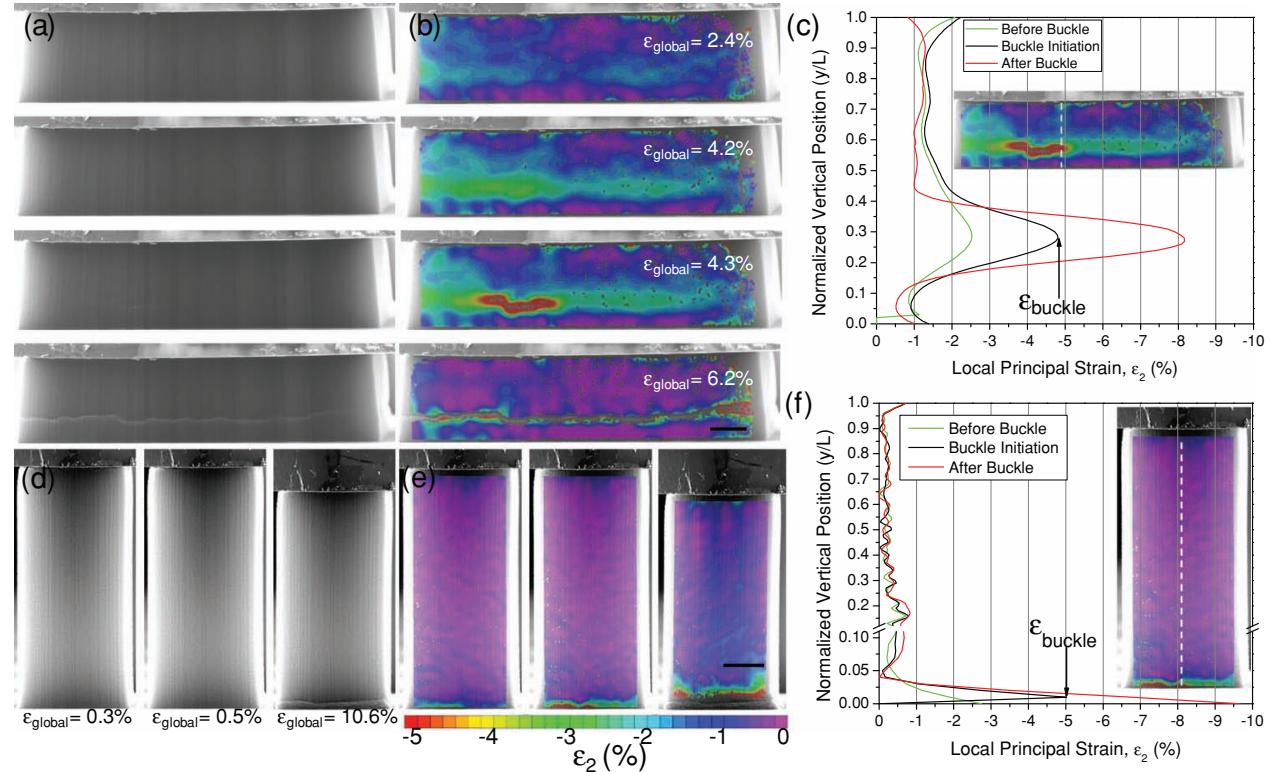

Figure 4. Crushing behavior of a-c) $100 \mu \mathrm{m}$ wide, 25 tall $\mu \mathrm{m}$ CNT array column and the bottom-up buckle accumulation of $d-f) 30 \mu m$ wide, $75 \mu \mathrm{m}$ tall CNT array column. Crushing behavior observed a) in the SEM image sequence originates near the column midsection and rapidly propagates throughout the width of the column, while b) strain maps and c) vertical profiles indicate that buckle formation and propagation occurs at approximately $5 \%$ local strain $\left(\varepsilon_{2}\right)$. The first and third buckle of the bottom-up buckle accumulation mode is observed in d) a representative SEM sequence. e) Strain maps show that strain is highly concentrated near the bottom of the column during buckle formation, while f) a vertical strain profile of the column obtained during initial buckle formation clearly indicates buckle formation and propagation at $5 \%$ local strain. Scale bars $=10 \mu \mathrm{m}$. Videos of the SEM deformation sequences and strain $\left(\varepsilon_{2}\right)$ evolution for the columns may be found in Video S4 and S6 in the Supporting Information. 


\subsubsection{Bottom-Up Buckle Accumulation of Intermediate Aspect Ratio Columns}

Intermediate aspect ratio columns develop and accumulate buckles strictly at their base and across the entirety of their widths by a mechanism characterized as bottom-up buckle accumulation. These columns include the nominally 50 and $75 \mu \mathrm{m}$ tall columns with 30 and $100 \mu \mathrm{m}$ widths, representing aspect ratios between approximately 0.6-2.7. During initiation of bottom-up buckling accumulation, a buckle originates near the growth substrate and propagates laterally across the entire width of the column. New buckles are added directly above the previous buckle in a periodic fashion, forming a collective buckling front that grows in height as buckles are added. These columns sway laterally during buckle accumulation, in sync with the propagation of each consecutive buckle. Greater than a dozen coordinated buckles may form prior to the buckling front overtaking the entirety of the column length. Previous studies of CNT array column mechanics have observed bottom-up buckle accumulation for cylindrical CNT array columns of similar aspect ratio; ${ }^{[13]}$ however, the crushing and bending modes have not been previously reported. A video of a $100 \mu \mathrm{m}$ wide, $75 \mu \mathrm{m}$ tall CNT array column undergoing bottom-up buckle accumulation may be viewed in video S5 in the Supporting Information.

The formation of the first and third buckle of a representative $30 \mu \mathrm{m}$ wide, $75 \mu \mathrm{m}$ tall CNT array column is displayed in Figure $4 d-f$ ). Formation of the first buckle for this column (propagating from left to right) occurs near the substrate at a global strain of $0.6 \%$. This buckling event resembles a smallscale collapse of the column in the region directly above the substrate. This buckling event is subtle and could easily be overlooked by SEM inspection without careful examination. Strain maps show that nearly all deformation occurs within the bottom $5 \%$ of the column height during formation of the first buckle, with buckle formation and propagation occurring at approximately $5 \%$ local strain. The vertical strain profile shown in Figure 4e, obtained at the leading edge of the buckle again confirms a local strain of $5 \%$, consistent with the previous deformation modes. Video S6 in the Supporting Information displays the deformation sequence and $\varepsilon_{2}$ evolution of the $30 \mu \mathrm{m}$ wide, $75 \mu \mathrm{m}$ tall CNT array column depicted in Figure 3a,b.

As with all columns of this deformation type, additional applied strain leads to the formation of additional buckles. The third sequential buckle, formed at $10.6 \%$ global strain, is displayed in the last frame of Figure $4 \mathrm{~d}$,e. This buckle is more readily identifiable than the first, resembling the inwardly folded structure of buckles observed for previously examined columns. Strain is well distributed in upper portion of the column after the formation of the third buckle, although generally at a magnitude of $0.75 \%$ or less. Near the top of the accumulated buckles, strain is locally enhanced, with a local magnitude of approximately 5\%. Image correlation for the accumulated buckles is generally lost below the buckling front as a result of the folded nature of the buckles. As a result, strain maps may only be generated at and above the most recently acquired buckle for these columns.

\subsection{Stress-Strain Behavior}

CNT array buckle formation and propagation observed by DIC analysis may also be correlated to the stress-strain behavior of the columns. The bottom-up buckling mode produces periodic stress-strain oscillations every $4-6 \mu \mathrm{m}$ of global vertical displacement, synchronized with the formation of consecutive buckles. The stress-strain behavior of a $30 \mu \mathrm{m}$ wide, $75 \mu \mathrm{m}$ tall column (separate from that examined in Figure $4 \mathrm{~d}-\mathrm{f}$ ) undergoing bottom-up buckle accumulation is presented in Figure 5a. Strain maps and vertical strain profiles are further evaluated at points 1-6 on the stress-strain curve, and are displayed in Figure 5b,c, respectively.

Strain maps obtained from points 1-5 present the complete initiation and propagation of a buckle from right to left at the bottom of the column, while the strain map at frame 6 is obtained near the maximum applied global strain of $17 \%$, after the formation of multiple buckles. The buckling event observed in points 1-5 leads to a decrease of the global applied stress and a negative differential stiffness during buckle propagation (Figure 5a). Strain maps during this period show a concentration of strain at the bottom of the pillar, with a principal strain of approximately $5 \%$ leading the propagating buckle. Vertical profiles of $\varepsilon_{2}$ obtained through the midsection of the column (Figure $5 \mathrm{c}$ ) indicate that strain in the upper portion of the column is less than $0.5 \%$ during the buckling event. The strain in this upper section of the column increases only $0.1 \%$ on average between frames 1-5, although global strain increased by approximately $1 \%$ during the same period. Near the maximum applied global strain of $17 \%$, observed in frame 6 , strain above the buckled region has increased significantly to approximately $1.5 \%$ and is relatively uniformly distributed. The continuous strain accumulation in the top portion of the column indicates that although most strain energy is utilized for the formation of buckles, approximately $10 \%$ of the applied global strain is retained within the unbuckled top region, estimated by comparing the average strain of the top portion of the column (Figure 5c) to the global strain value.

Strain maps generated by DIC clearly identify the locations of greatest strain and buckle initiation. However, factors based upon CNT array morphology that may contribute to their local mechanical response are not fully understood. Undoubtedly, the mechanical properties of CNT arrays depend on their density. The average density of CNT arrays grown by our process is approximately $15 \mathrm{mg} / \mathrm{cm}^{3}$, or $\approx 1 \%$ relative to an ideal tightly packed array. Specifically, the location of buckle initiation may depend on morphological variations within the column, including variations in CNT bundling, alignment, and density. These variations result from the collective population-based nature of the CNT growth process. For example, we find that the density of CNT arrays decreases gradually as growth proceeds and individual catalyst particles deactivate, so the density is typically lowest at the bottom of the array. In separate work, we have confirmed these density trends by mass measurements and X-ray absorption. ${ }^{[30]}$ A lower density portion of the array would experience a higher load per CNT, thus resulting in a higher local stress concentration. Similarly, the orientation of the CNTs into cellular arrangements with variable geometries and strut thicknesses may lead to differences in the stress- 

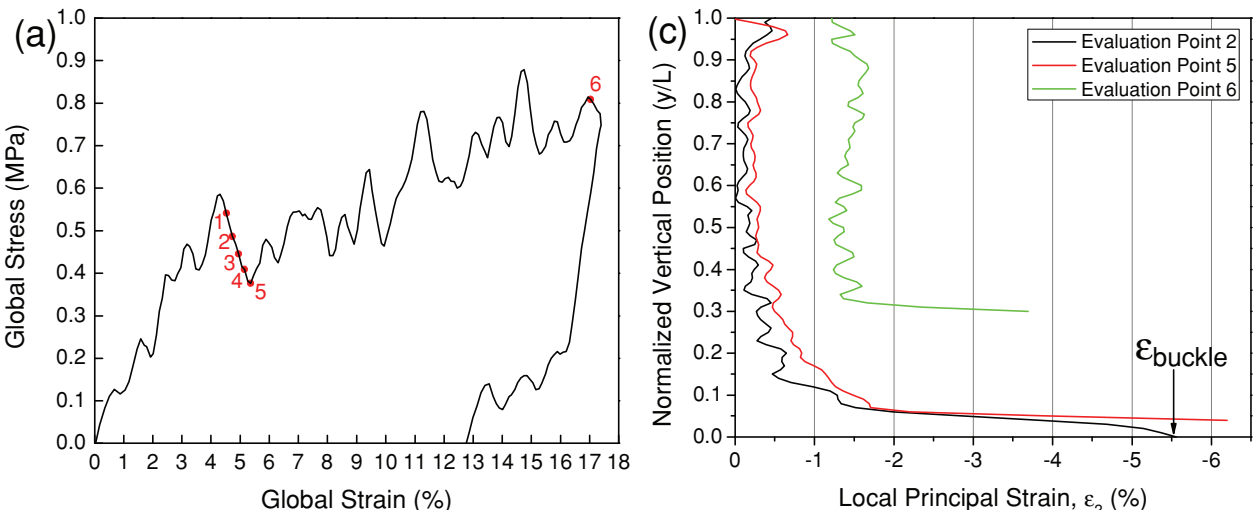

(b)

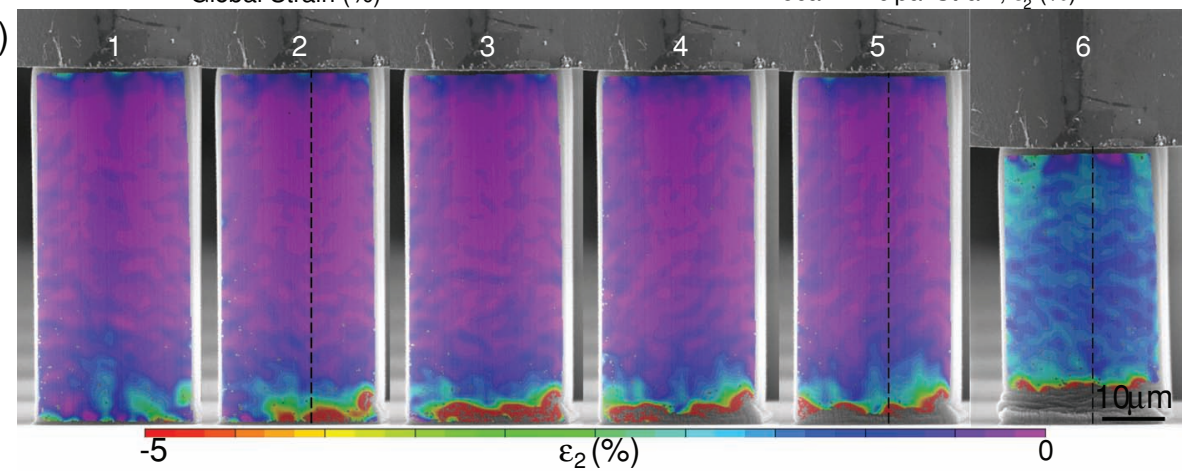

Figure 5. Stress and strain evolution for a $75 \mu \mathrm{m}$ tall, $30 \mu \mathrm{m}$ wide column exhibiting bottom-up buckle accumulation. The evaluation points $1-5$ displayed on a) the global stress-strain plot represent the formation and propagation of a CNT array buckle, while point 6 represents a state near the maximum applied global strain. b) Sequential $\varepsilon_{2}$ maps analyzed at these points reveal strain concentration at point 1 , followed by complete buckle propagation from right to left in points $2-5$. The strain field near maximum applied global strain reveals relatively uniform strain distribution throughout a majority of the unbuckled portion on the column, with enhanced strain near the buckle accumulation zone. c) Vertical strain profiles, obtained about the vertical dotted lines in frames 2, 5, and 6 show buckle formation at approximately $5 \%$ local strain and the gradual increase in strain magnitude in the unbuckled portion of the column.

strain response at a local level. Unfortunately, precise measurement of the density of individual CNT columns by X-ray or optical absorption is beyond the scope of this work, and suffers tradeoffs between the small beam spot required for spatial mapping and the need for a sufficiently large number of CNTs for signal collection. Nevertheless, the results herein show that certain consistent observations about column mechanics can be made in spite of possible density and orientation variations.

\section{Conclusions}

By using DIC to track the deformation of CNTs within an array, the spatially resolved mechanics of compressed CNT arrays was explored for the first time. No surface preparation was required to generate a traceable speckle pattern for DIC analysis. Rather, the high-contrast pattern established by the CNT array during SEM imaging was found to be suitable for DIC analysis and was stable during significant local strain. Deformation modes of CNT array columns were systematically altered by varying column dimensions, with each mode producing significant differences in the location and behavior of CNT array buckling. Strain within the arrays is distributed non-uniformly for all columns, with CNT buckling initiating only in areas of greatest localized strain. Furthermore, we found that a local $\varepsilon_{2}$ magnitude of approximately $5 \%$ is a universal CNT array buckling criterion for the CNT array columns examined in this study, in spite of the significantly different deformation behaviors observed at the column level. Strain away from the buckled region was always less than 5\%. Additional testing and analysis is required to determine how this observed buckling criterion varies between CNT arrays of significantly different morphologies. Nevertheless, the DIC technique provides a rapid, noncontact means to measure full-field deformation of CNT arrays and can serve as a quantitative platform for investigating the hierarchical mechanics of CNT arrays and related materials.

\section{Experimental Section}

In Situ Compression: In situ SEM compression was enabled using a custom-built micromechanical test frame within an FEI Quanta ESEM. ${ }^{[10]}$ Compression of all columns having a width of 10 or $30 \mu \mathrm{m}$ utilized a $40 \mu \mathrm{m} \times 40 \mu \mathrm{m}$ diamond flat punch, while the $100 \mu \mathrm{m}$ wide columns utilized a $125 \mu \mathrm{m}$ diameter sapphire flat punch. The test frame stage allows for sample positioning with three degrees of freedom using piezoelectric actuation. Displacement data and image acquisition were acquired using an external PC running National Instruments LabVIEW 
and 4Pi Revolution imaging software, respectively. Secondary electron images were acquired between indenter tip displacement increments of between 5-500 nm. Typical image resolutions ranged from 2000 3000 pixels per line and were acquired at a $10 \mu \mathrm{s}$ pixel $^{-1}$ scan rate. Images were acquired in 16-bit grayscale format.

Digital Image Correlation: SEM images acquired from in situ CNT array compression experiments were analyzed using VIC-2D commercial software (Correlated Solutions, Inc.). Typical DIC conditions include 25 to 50-pixel evaluation subsets at 3-5 pixel steps. The low-pass filter option was utilized to minimize the influence of image noise. The correlation algorithm utilized a 4-tap spline gray value interpolation. A normalized squared differences method was selected to accommodate potential changes in grayscale from image to image. The maximum prediction margin was 0.02 pixels at a 0.1 pixel maximum confidence interval.

CNT Growth: CNT array columns were fabricated utilizing photolithographically defined thin film catalyst $\left(1 / 10 \mathrm{~nm} F e / \mathrm{Al}_{2} \mathrm{O}_{3}\right)$ on silicon wafer substrates coated with a $300 \mathrm{~nm} \mathrm{SiO} 2$ layer. After patterning and catalyst deposition, the substrates were heated to $775{ }^{\circ} \mathrm{C}$ in a horizontal tube furnace (22 $\mathrm{mm}$ inner diameter) for 10 minutes, under a flow of $100 / 400 \mathrm{sccm}$ of $\mathrm{H}_{2} / \mathrm{He}$. This condition was maintained for 10 more minutes, and then $100 \mathrm{sccm}$ of $\mathrm{C}_{2} \mathrm{H}_{4}$ was introduced. The height of the CNT columns was determined by the duration of $\mathrm{C}_{2} \mathrm{H}_{4}$ flow, and the vertical CNT column growth rate was approximately $50 \mu \mathrm{m} \mathrm{min}{ }^{-1}$. A series of samples was grown to heights of 25,50 , and 75 $\mu \mathrm{m}$ by varying the growth time from 0.5 to $1.5 \mathrm{~min}$. Square columns with widths of 10,30 , and $100 \mu \mathrm{m}$ were evaluated in this study. The columns comprise multiwall CNTs with diameters ranging from 8-10 nm $( \pm 1 \sigma)$, as previously determined by TEM and X-ray scattering. ${ }^{[30]}$

\section{Supporting Information}

Supporting Information is available from the Wiley Online Library or from the author.

\section{Acknowledgements}

The authors gratefully acknowledge Dr. Robert Wheeler (AFRL/RX MicroTesting Solutions LLC) for assistance with in situ data collection. M.R.M. and J.W.B. acknowledge financial support from the Air Force Office of Scientific Research (AFOSR), Dr. Byung-Lip (Les) Lee, Program Manager. S.J.P. and A.J.H. acknowledge support of the National Science Foundation (CMMI-0927364) and the AFOSR Young Investigator Program (11-NA-018), Dr. Byung-Lip (Les) Lee, Program Manager.

Received: March 12, 2012

Revised: May 7, 2012

Published online: July 20, 2012

[1] J. Xu, T. S. Fisher, Int. J. Heat Mass Transfer 2006, 49, 1658

[2] B. Cola, J. Xu, C. Cheng, X. Xu, T. Fisher, H. Hu, J. Appl. Phys. 2007, 101, 054313.

[3] B. A. Cola, J. Xu, T. S. Fisher, Int. J. Heat Mass Transfer 2009, 52, 3490.

[4] M. Park, B. A. Cola, T. Siegmund, J. Xu, M. R. Maschmann, T. S. Fisher, H. Kim, Nanotechnology 2006, 17, 2294.
[5] O. Yaglioglu, J. Hart, R. Martens, A. Slocum, Rev. Sci. Instrum. 2006 77, 095105

[6] T. Yamada, Y. Hayamizu, Y. Yamamoto, Y. Yomogida, A. Izadi-Najafabadi, D. N. Futaba, K. Hata, Nat. Nanotechnol. 2011, 6, 296.

[7] G. Toth, J. Mäklin, N. Halonen, J. Palosaari, J. Juuti, H. Jantunen, K. Kordas, W. G. Sawyer, R. Vajtai, P. M. Ajayan, Adv. Mater. 2009, 21, 2054.

[8] S. Pathak, G. Cambaz, S. Kalidindi, G. Swadener, Y. Gogotsi, Carbon 2009, 47, 1969.

[9] T. Tong, Y. Zhao, L. Delzeit, A. Kashani, M. Meyyappan, A. Majumdar, Nano Lett. 2008, 8, 511.

[10] M. R. Maschmann, Q. Zhang, R. Wheeler, F. Du, L. Dai, J. Baur, ACS Appl. Mater. Interfaces 2011, 3, 648.

[11] M. R. Maschmann, Q. Zhang, F. Du, L. Dai, J. Baur, Carbon 2011, 49, 386.

[12] A. Cao, P. L. Dickrell, W. G. Sawyer, M. N. Ghasemi-Nejhad P. M. Ajayan, Science 2005, 310, 1307.

[13] S. B. Hutchens, L. J. Hall, J. R. Greer, Adv. Funct. Mater. 2010, 20 2338.

[14] A. A. Zbib, D. Mesarovic, E. T. Lilleodden, D. McClain, J. Jiao, D. F. Bahr, Nanotechnology 2008, 19, 175704.

[15] P. D. Bradford, X. Wang, H. Zhao, Y. T. Zhu, Carbon 2011, 49, 2834.

[16] S. B. Hutchens, A. Needleman, J. R. Greer, J. Mech. Phys. Solids 2011, 59, 2227

[17] A. F. Bastawros, H. Bart-Smith, A. G. Evans, J. Mech. Phys. Solids $2000,48,301$

[18] H. Bart-Smith, A. F. Bastawros, D. R. Mumm, A. G. Evans, D. J. Sypeck, H. N. G. Wadley, Acta Mater. 1998, 46, 3583.

[19] H. Jin, W.-Y. Lu, S. Scheffel, T. D. Hinnerichs, M. K. Neilsen, Int. J. Solids Struct. 2007, 44, 6930.

[20] J. Zhou, Z. Gao, A. M. Cuitino, W. O. Soboyejo, Mater. Sci. Eng. A 2004, 386, 118.

[21] M. A. Sutton, J. J. Orteu, H. W. Schreier, Image Correlation for Shape, Motion, and Deformation Measurements. Basic Concepts, Theory and Applications, Springer Science+Business Media, New York 2009.

[22] D. S. Gianola, A. Sedlmayr, R. Mönig, C. A. Volkert, R. C. Major, E. Cyrankowski, S. A. S. Asif, O. L. Warren, O. Kraft, Rev. Sci. Instrum. 2011, 82, 063901

[23] B. G. Clark, D. S. Gianola, O. Kraft, C. P. Frick, Adv. Eng. Mater. 2010, 12, 808.

[24] W. Scrivens, Y. Luo, M. Sutton, S. Collette, M. Myrick, P. Miney, P. Colavita, A. Reynolds, X. Li, Exp. Mech. 2007, 47, 63.

[25] N. Li, M. Sutton, X. Li, H. Schreier, Exp. Mech. 2008, 48, 635.

[26] J. H. Warner, N. P. Young, A. I. Kirkland, G. A. D. Briggs, Nat. Mater. 2011, 10, 958

[27] M. Sutton, N. Li, D. Garcia, N. Cornille, J. Orteu, S. McNeill, H. Schreier, X. Li, A. Reynolds, Exp. Mech. 2007, 47, 789.

[28] M. Sutton, N. Li, D. Joy, A. Reynolds, X. Li, Exp. Mech. 2007, 47, 775.

[29] M. A. Sutton, J. H. Yan, V. Tiwari, H. W. Schreier, J. J. Orteu, Opt Lasers Eng. 2008, 46, 746.

[30] M. Bedewy, E. R. Meshot, M. J. Reinker, A. J. Hart, ACS Nano 2011, 8974.

[31] H. Bruck, S. McNeill, M. Sutton, W. Peters, Exp. Mech. 1989, 29, 261. 